\title{
Occurrence of thermophilic Campylobacter spp. contamination on vegetable farms in Malaysia.
}

\begin{abstract}
The aim of the present study was to examine the prevalence of thermophilic Campylobacter spp. (Campylobacter jejuni and Campylobacter coli) in soil, poultry manure, irrigation water, and freshly harvested vegetables from vegetable farms in Malaysia. C. jejuni was detected in $30.4 \%$ and $2.7 \%$ of the soil samples, $57.1 \%$ and $0 \%$ of the manure samples, and $18.8 \%$ and $3 \%$ of the vegetable samples from farm $\mathrm{A}$ and farm $\mathrm{B}$, respectively, when using the MPNPCR method. Campylobacter spp. was not found in any of the irrigation water samples tested. Therefore, the present results indicate that the aged manure used by farm A was more contaminated than the composted manure used by farm B. Mostly, the leafy and root vegetables were contaminated. C. coli was not detected in any of the samples tested in the current study. Both farms tested in this study were found to be contaminated by campylobacters, thereby posing a potential risk for raw vegetable consumption in Malaysia. The present results also provide baseline data on Campylobacter contamination at the farm level.
\end{abstract}

Keyword: Campylobacter; Prevalence; Pre-harvest; Vegetable. 